\title{
28 Research Suare \\ Generating a New Chaotic System Using Two Chaotic Rossler-Chua Coupling Systems
}

Ryam Salam Abdulaali

University of Baghdad Al-Jaderyia Campus College of Science

Raied K. Jamal

University of Baghdad Al-Jaderyia Campus College of Science

Salam K. Mousa ( $\nabla$ salam.khalaf@uoanbar.edu.iq )

University of Anbar

\section{Research Article}

Keywords: Chaos, Nonlinear dynamic, Chua system, Rössler system, Coupling system, Attractor, Time series, FFT.

Posted Date: July 9th, 2021

DOl: https://doi.org/10.21203/rs.3.rs-672916/v1

License: (c) (i) This work is licensed under a Creative Commons Attribution 4.0 International License. Read Full License

Version of Record: A version of this preprint was published at Optical and Quantum Electronics on November 2nd, 2021. See the published version at https://doi.org/10.1007/s11082-021-03341-9. 


\section{Abstract}

It is proposed in this paper that a new chaotic system may be formed by combining two distinct chaotic systems, such as the Rossler system and the Chua system, in which the $x$ dynamic of the Rossler system is linked with the $z$ dynamic of the Chua system, results in a new chaotic system. Some of the basic dynamic behavior is explored and examined for new system by using the Matlab program. They noticed that it was a difference in the time series of the Chua system and this in turn led to a difference in the attractor, as the attractor of the Chua system changed from double scroll to single scroll and this led to change of the bandwidth of the Chua system, meaning that the Rössler system affected the Chua system, which led to an increase in the possibility of using this system in secret communications.

\section{Introduction}

In the twentieth century, there were many scientific revolutions that improved our knowledge of the nature of unpredictable behavior. One of these revolutions was the study of chaos in dynamics. Chaos Theory is the theory that deals with nonlinear dynamic systems, which exhibits behavior as if it were random, and that behavior results either by the inability to determine the initial conditions or by the potential physical nature of quantum mechanics. So, Chaos is a characteristic which we stick it to the systems that have unexpected long-term future [1-2]. Any dynamic system (variable over time) is highly sensitive to initial conditions; it means that the future of this system will be very dependent and greatly affected by its current state and condition. The small changes in current circumstances can lead to completely different results in the distant future, so the shape of the system is changing completely and be very difficult to predict. The simple change in the values of the primary system variables has a far greater effect than that initial change in the end result, due to other factors and variables in the system, these systems are very sensitive to any change in the initial values or variables whatever the change values are small [3-4]. Many academics are interested in the study of nonlinear dynamics and chaos theory, and they are working on developing mathematical models that mimic the fields of nonlinear chaotic physical systems. Nonlinear phenomena may be found in almost every area of study, including engineering, physics, chemistry, biology, economics, and social science. Nonlinear chaotic systems are used in a variety of applications, including planetary climate prediction models, neural network models, data compression, turbulence, nonlinear dynamical economics, information processing, preventing the collapse of power systems, high-performance circuits and devices, and liquid mixing with minimal power consumption [57]. lin this work, the effect of coupling the variable $x$ of the Rössler system with the variable $z$ of the Chua system and studying the behavior of the chaotic system resulting from this coupling was studied.

\section{Chua's And Rössler Circuits}

Chua's "double scroll" dynamical system was the first chaotic system to be seen in the laboratory, verified by computer simulation, and rigorously mathematical backed up by rigorous mathematical proof [8-10]. Originally created as an electric circuit, the Chua system (also called Chua circuit) has the following three dimensionless equation representations: 


$$
\left\{\begin{array}{c}
x_{1}^{\prime}=\alpha\left[y_{1}-x_{1}-g(V)\right] \\
y_{1}^{\prime}=x_{1}-y_{1}+z_{1} \\
z_{1}^{\prime}=-\beta y_{1} \\
g(V)=c_{1} x_{1}+\frac{1}{2}\left(d-c_{1}\right)\left[\left|x_{1}+1\right|-\left|x_{1}-1\right|\right]
\end{array}\right.
$$

Where the parameters $, \beta, c_{1}$, and $d$ were $15.05,25.58,-0.7142857,-1.142$ respectively, while the initial condition $\left[\mathrm{x}_{1}, \mathrm{y}_{1}\right.$, and $\left.\mathrm{z}_{1}\right]$ were $[1.6,0,1.6]$ respectively.

A Rössler model was also proposed in order to have the simplest attractor with chaotic behavior that does not have the feature of symmetrical behavior. There are three non-linear ordinary differential equations in the Rössler model, which describe a continuous-time nonlinear system that exhibits chaotic behavior, which may be explained by the fractal features of the attractor. The Rossler system may be described using the three-dimensionless equation [11] that is linked to differential equations:

$$
\left\{\begin{array}{c}
x_{2}^{\prime}=-\left(y_{2}+z_{2}\right) \\
y_{2}^{\prime}=x_{2}+a y_{2} \\
z_{2}^{\prime}=b+x_{2} z_{2}-c_{2} z_{2}
\end{array}\right.
$$

These differential equations describe a continuous-time dynamical system that displays chaotic dynamics related with the fractal characteristics of the attractor, as defined by these differential equations. It have seven term, one quadratic nonlinearity and three parameter, where ${ }^{a, b, c_{2}} \in R_{3}$, and they are dimensionless parameters, the three variables that change as time progresses are denoted by $x 2$, $y 2$, and $z 2$ respectively Otto begins by examining the values of actual parameters in the real world. According to $E$. Rössler, if $a$ and $b$ are equal to 0.2 and $c 2$ equal to 5.7, the system displays chaotic behavior. Each of the first two equations contains linear terms that cause oscillations in the variables $\mathrm{x}$ and $y$ to be generated. Because the final equation has just one nonlinear term (xz), the system exhibits the chaotic behavior that has been predicted. The initial conditions $[x 2, y 2$, and $z 2]$ were $[1,1,0]$ respectively.

The Rössler and Chua systems are disaccorded components, so the signal that drive out from Rössler system \& drive in Chua system will be affecting in behavior of the second system. To employ new chaotic scheme, contact two chaotic systems as seen in Figure (1). The Rössler system consists of three channels can use any channels to couple with Chua circuit. Now when the two circuit (R ssler-Chua circuits) are coupled (i.e. the output of variable of Rössler system was coupled with variable of Chua system, as shown in Figure (1). The Rössler and Chua systems are disaccorded components, so the signal that drive out from Rössler system and drive in Chua system will be affecting in behavior of the second system. The dimensionless states equations of Chua circuit with coupled will be [12]: 


$$
\left\{\begin{array}{c}
x_{1}^{\prime}=\alpha\left(y_{1}-x_{1}-g\left(x_{1}\right)\right)-\left(y_{2}+z_{2}\right) \\
y_{1}^{\prime}=x_{1}-y_{1}+z_{1} \\
z_{1}^{\prime}=-\beta y_{1} \\
g(V)=c_{1} x_{1}+\frac{1}{2}\left[\left(d-c_{1}\right)\left(\left|x_{1}+1\right|-\left|x_{1}-1\right|\right)\right]
\end{array}\right.
$$

\section{Results And Discussion}

In this paper, the behavior of the new chaotic system was studied numerically by programming the three differential equations of Chua and Rössler (equations 1, 2, and 3) using the Matlab program, where the differential equations were solved using Runga-Kutta integration of the fourth degree. The time series of the Rössler system shown in Figure (2), where represent the output voltage in $x 2$-dynamics, where the output signal in a range +10 to -10 , while the time range is 0 to 150 a.u. with interval time of 0.01 a.u. Figure (3), is representing the time series of three state variable $x 2, y 2$ and $z 2$ of Chua's system. The Figures (4), (5), and (6) that shows double-scroll strange attractor mode that represents the strange attractors of pairs $\left(\mathrm{y}_{1}-\mathrm{x}_{1}\right),\left(\mathrm{z}_{1}-\mathrm{x}_{1}\right)$, and $\left(\mathrm{z}_{1}-\mathrm{y}_{1}\right)$ respectively, that shows also double-scroll strange, and Figure (7) represent the strange attractor in three dimension $\left(z_{1}-y_{1}-x_{1}\right)$. When the variable $x_{2}$ of the Rössler system is coupled with variable $z_{1}$ for a Chua system, the time series of Chua circuit will change to new behavior and become as shown in Figure(8), where it is completely different in first state. The range values of output voltage are changing as result this coupled. Moreover the attractor of Chua system is showing "double scroll" pattern this behavior convert to homoclinic chaotic by coupled $\mathrm{x}_{2}$-dynamic of Rössler system with $z_{1}$-dynamic of Chua system, where Figures (9), (10), and (11) as shows strange attractors in pairs $\left(\mathrm{y}_{1}-\mathrm{x}_{1}\right),\left(\mathrm{z}_{1}-\mathrm{x}_{1}\right)$, and $\left(\mathrm{z}_{1}-\mathrm{y}_{1}\right)$ dynamics of Chua system with new scheme respectively. Figure (12) shows the strange attractors in three dimension $\left(\mathrm{z}_{1}-\mathrm{y}_{1}-\mathrm{x}_{1}\right)$ dynamics of Chua system with new scheme. Table (1) represent $x 1, y 1$, and $z 1$ dynamics ranges before and after coupling, where the dynamics of Chua system will increse after coupling.

Through Figures (13) and (14), the values of the ranges of the chaotic dynamical systems, which play an important role especially in the applications of covert and secure communication and which is clearly illustrated by performing Fourier transforms (FFT) of the chaotic dynamics before and after coupling has been observed. Chaotic systems are believed to have a much wider applicability with higher dimensional attractors. Several previous investigations are in agreement with the results obtained, despite the use of other chaotic systems different from those discussed in this investigation [13-14].

\section{Conclusion}

This work explains how to create a new and powerful chaotic system by using other chaotic systems and merging them. The change of the Chua system attractor, which was shown in the form of double scroll then converted to homoclinic chaotic by coupled $\mathrm{x}_{2}$-dynamic of Rössler circuit with $\mathrm{z}_{1}$-dynamic of Chua's 
circuit. In the FFT figures, it is noticed that the dynamic bandwidth $\mathrm{z}_{1}$-coupled has become more broad than it is in the case of non-coupling, and this point is very important in the subject of secret communications. Thus, the characteristic frequency of the Chua system has completely disappeared within the wide range formed as a result of the coupling, so the dynamic $z$-dynamic is more important than the other dynamics due to its discontinuous exponential dicey distribution.

\section{References}

1. J . E.Marsdenetal.,Texts in Applied Mathematics 2 Introduction to Applied Nonlinear Dynamical Systems and Chaos. 2003.

2. B. L. Bechtold and B. L. Bechtold, “Chaos theory as a model for strategy development," 2006.

3. S.Wigginsetal.,Introduction to Applied Nonlinear Dynamical Systems and Chaos. 2003.

4. D. S. Theories, “Dynamic Systems Theories,” pp. 258-312.

5. G. Chen and X. Dong, "From Chaos to Order: Method- ologies, Perspectives and Applications," World Scientific.

6. K. M. Cuomo, A. V. Oppenheim and S. H. Strogatz, "Synchronization of Lorenz-Based Chaotic Circuits with Applications to Communications," IEEE Transactions on Circuits and Systems-II: Analog and Digital Signal Proc-essing, Vol. 40, No. 10, 1993, pp. 626-633.

7. E. N. Lorenz, "Deterministic Nonperiodic Flow," Journal of Atmospheric Sciences, Vol. 20, No. 2, 1963, pp. 130-141. (1963)

8. CHUA, L. O., KOMURO, M., AND MATSUMOTO T., The double scroll family, IEEE Trans. Circuit and Systems, 31, (1986), 1072-1118.

9. MATSUMOTO, T. ,CHUA, L. O. , ANDKOMURO, M. , The Double Scroll , IEEE Trans .Circuit and Systems, 32, (1985), 797-818.

10. MATSUMOTO, T., CHUA, L. O. , AND AYAKI, K. , Reality of Chaos in the Double Scroll Circuit: A Comuter-Assisted Proof, IEEE Trans. Circuit and Systems, 35, (1988), 909-925.

11. Stephen Lynch. 2004. "Dynamical systems with application using Matlab",Birkhauser,2004.

12. R. K. Jamal and A. H. Mohamed. 2017. "Secure communication by using Chua's model", International Journal of ChemTech Research, 10(2017):739-749.

13. Raied K. Jamal , and Dina A. Kafi. 2019. "Secure Communication Coupled semiconductor Laser Based on Rössler Chaotic Circuits", IOP Conf. Series : Materials Science and Engineering,571 (2019):1-9.

14. Salam K. Mousa, and Raied K. Jamal. 2021. "Realization of a novel chaotic system using coupling dual chaotic system", Optical and Quantum Electronics, 53(2021):1-9.

\section{Tables}

Table (1): $x 1, y 1$, and $z 1$ dynamics ranges before and after coupling 


\begin{tabular}{|llll|}
\hline dynamics & $\mathrm{x} 1$ & $\mathrm{y} 1$ & $\mathrm{z} 1$ \\
\hline Before coupling & $-2.4: 2.4$ & $-0.4: 0.4$ & $-3.5: 3.5$ \\
\hline After coupling & $-3: 2$ & $-0.6: 0.6$ & $-4: 5$ \\
\hline
\end{tabular}

Figures

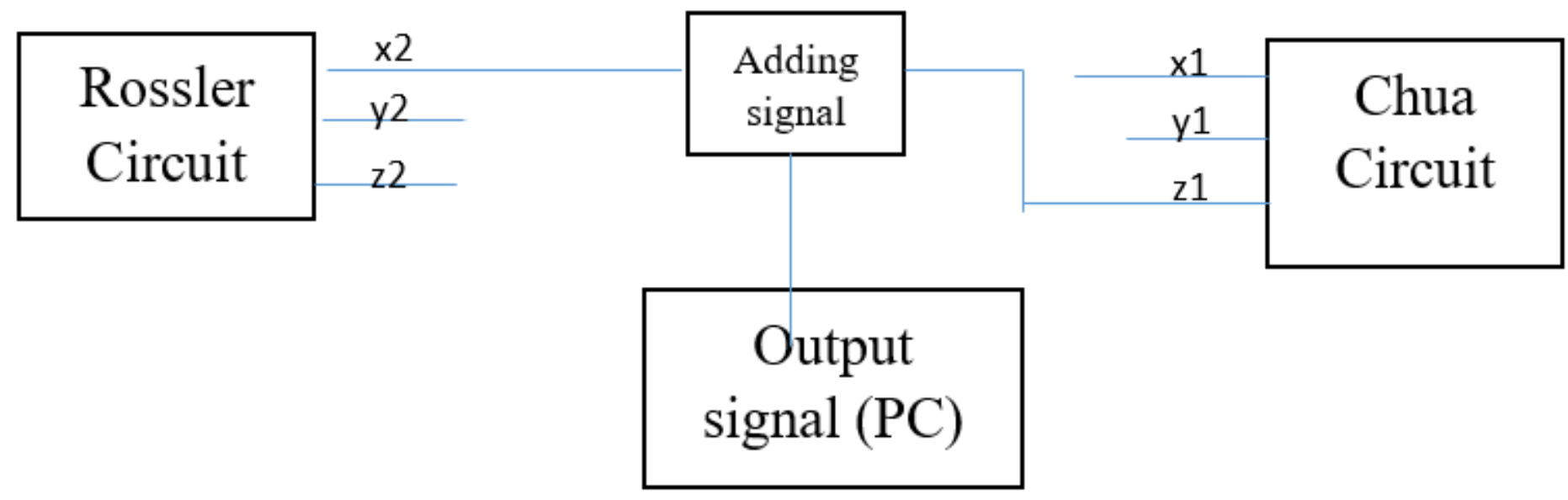

Figure 1

New chaotic dynamic scheme.

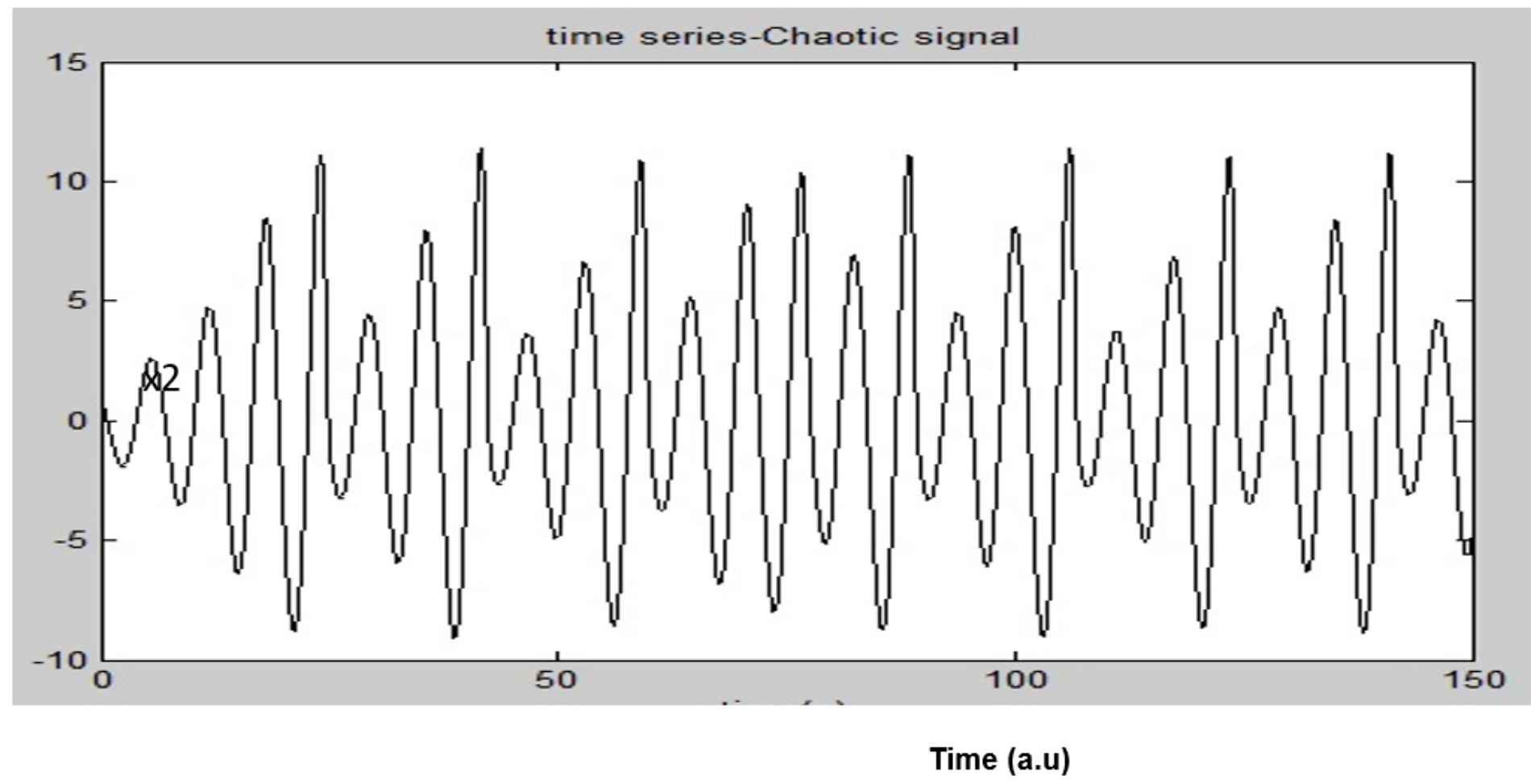

Figure 2 
Time series of x2-dynamics of Rössler system, where the parameters $a$ and $b=0.2$ and c_2 $=5.7$, while the initial condition $[x 2, y 2$, and $z 2]$ were $[1,1,0]$ respectively.

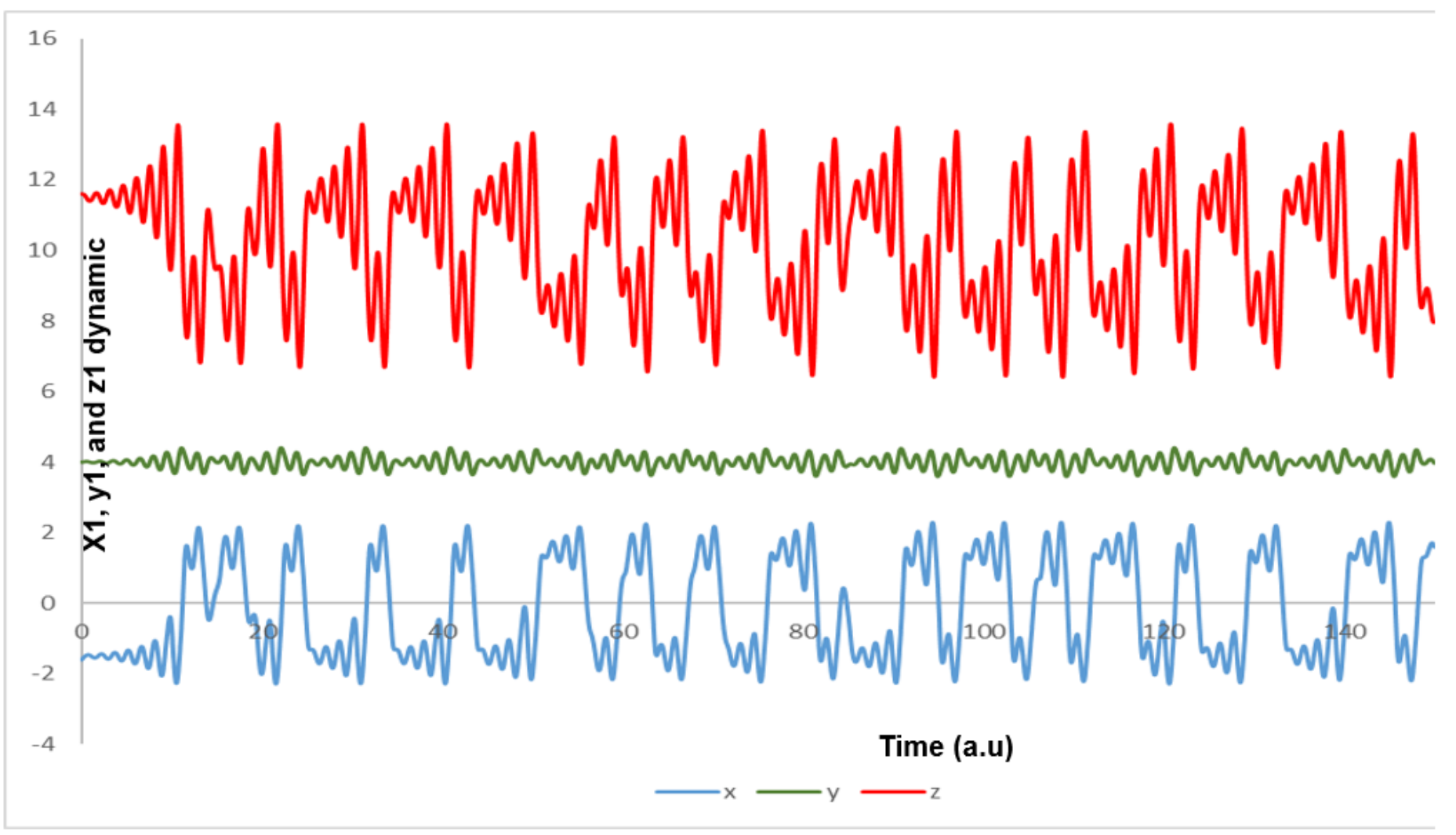

Figure 3

Time series of $x 1, y 1$, and $z 1$ - dynamics of Chua system, where the parametersa, $\beta, c 1$, and d were 15.05 , $25.58,-0.7142857,-1.142$ respectively, while the initial condition $[x 1, y 1$, and $z 1]$ were $[1.6,0,1.6]$.

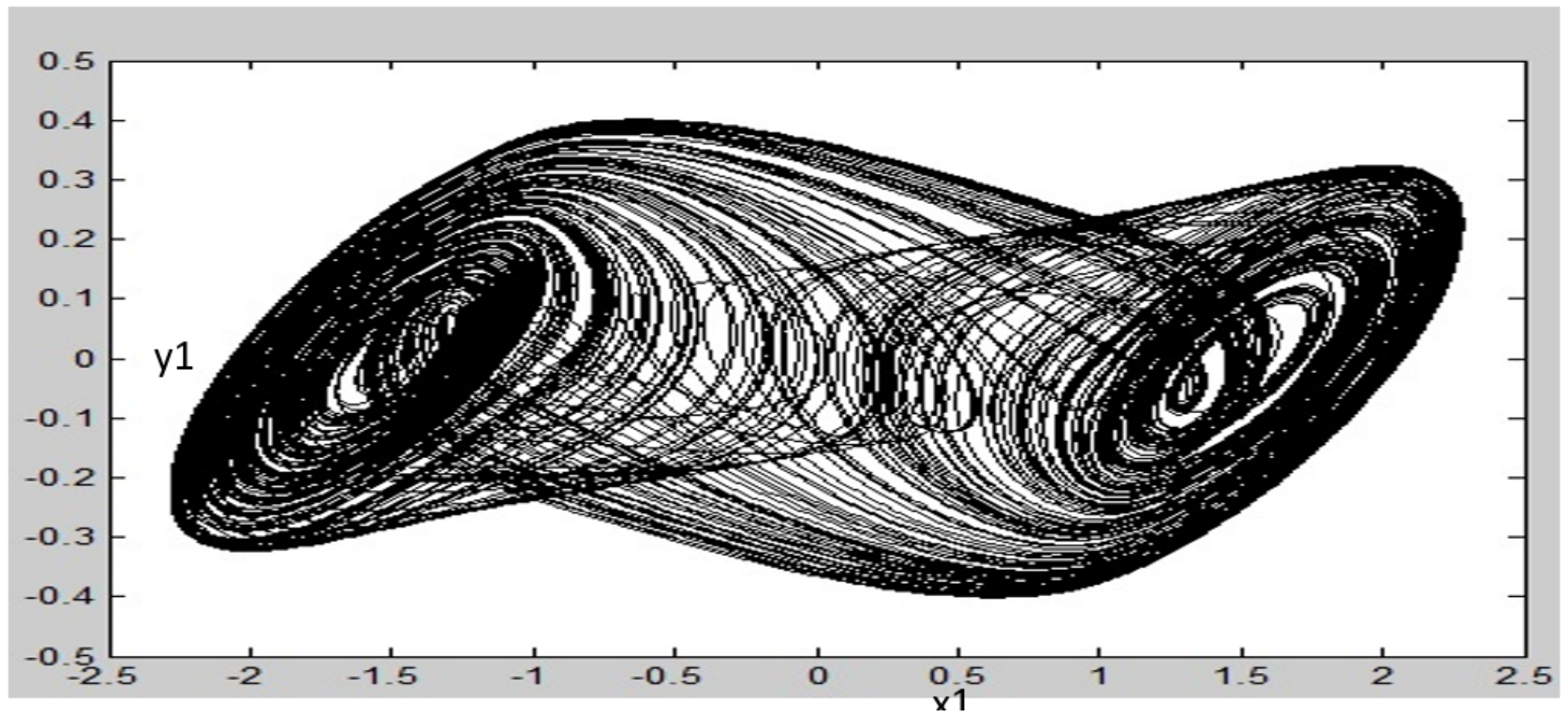

Figure 4 
Strange attractor in (y1-x1) dynamics of Chua system.

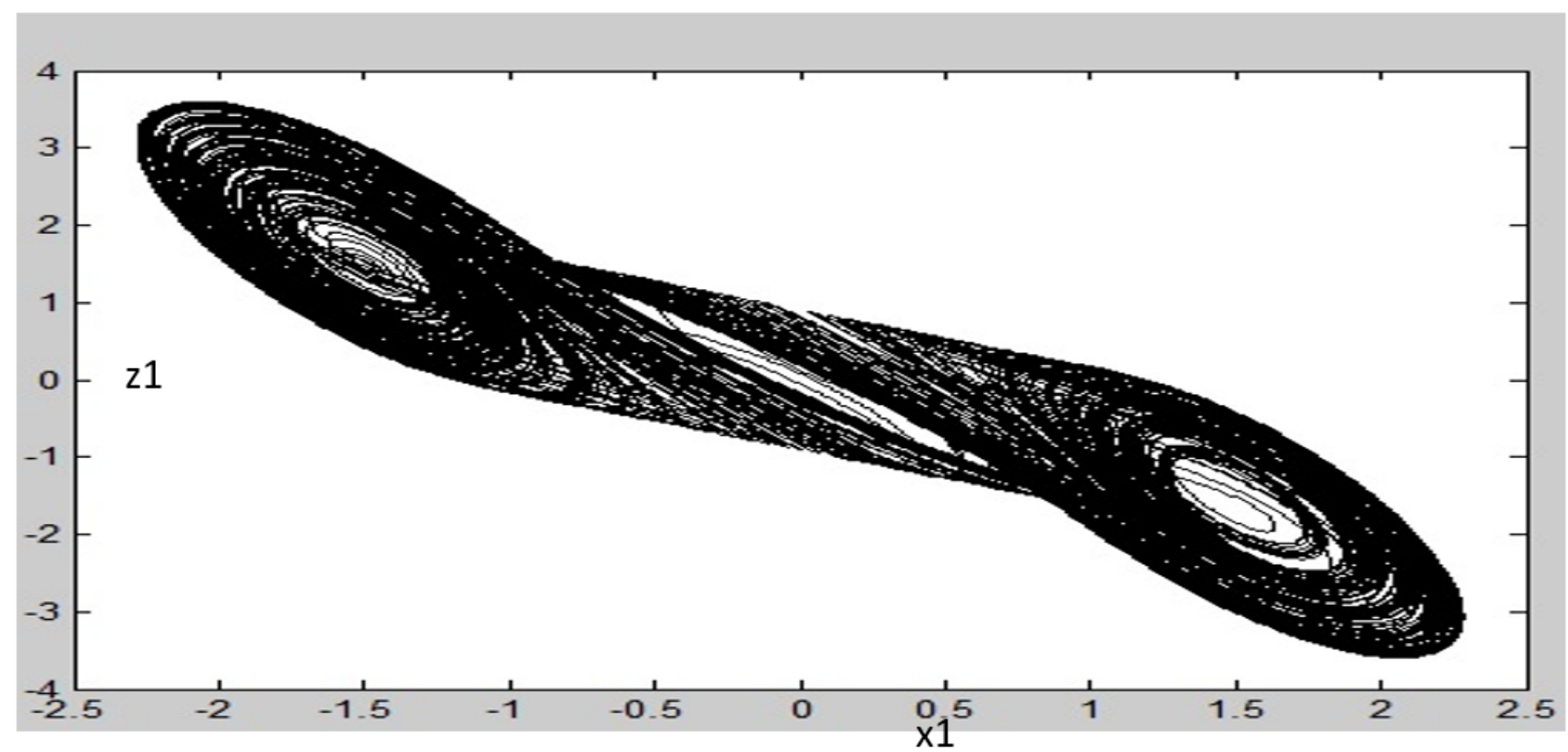

Figure 5

Strange attractor in (z1-x1) dynamics of Chua system.

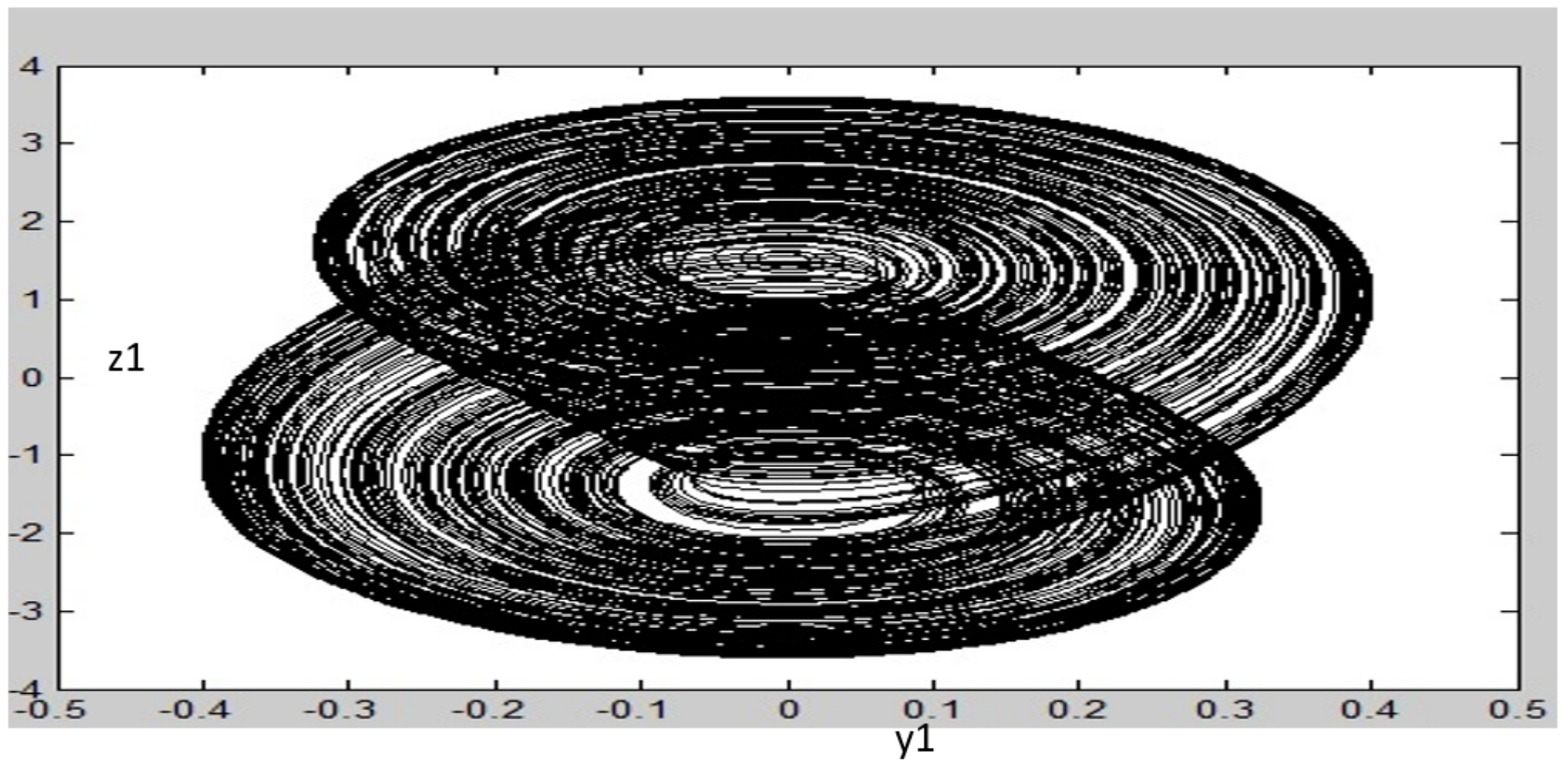

Figure 6

Strange attractor in (z1-y1) dynamics of Chua system. 


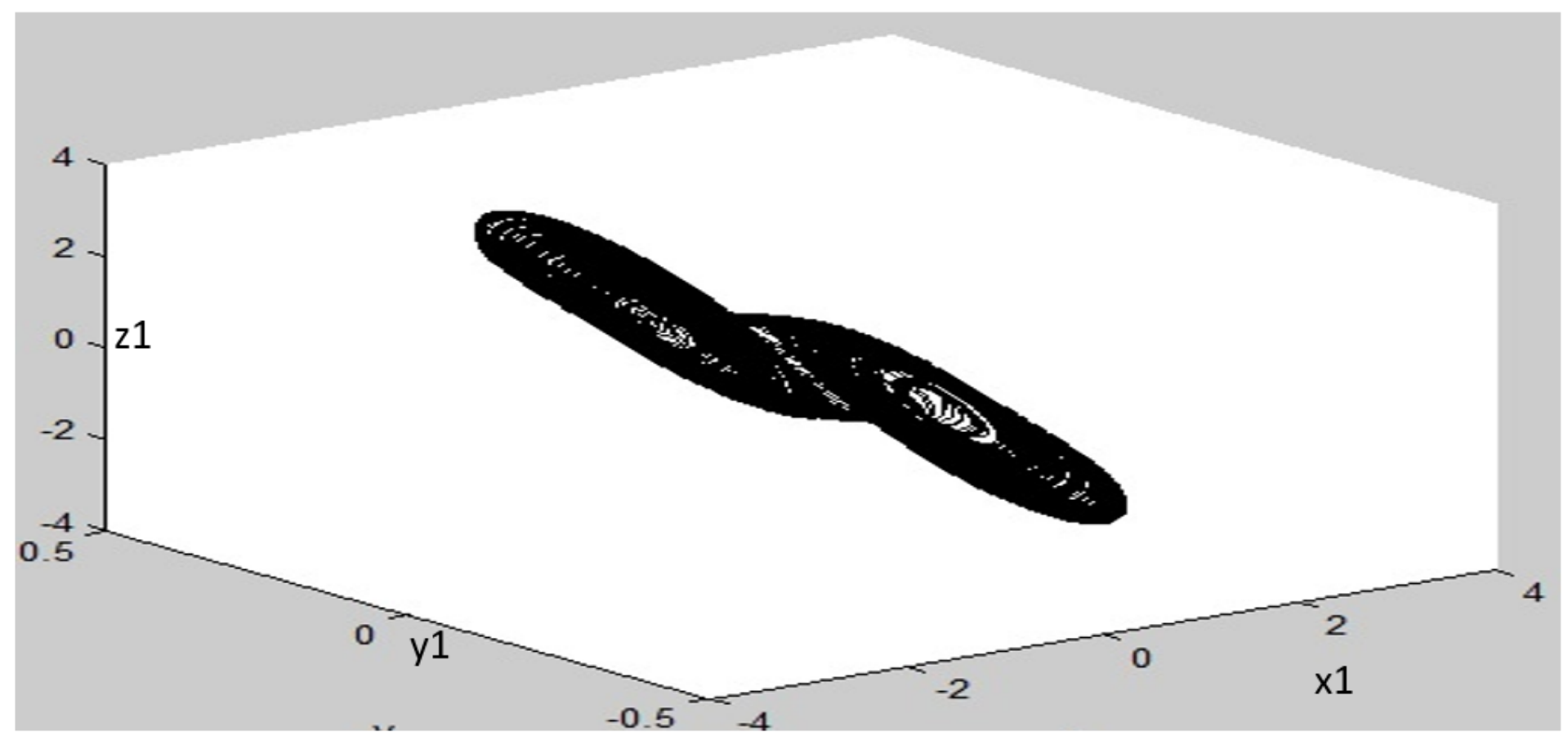

Figure 7

Strange attractor in 3D spaces (z2-y2-x2) dynamics of Chua system.

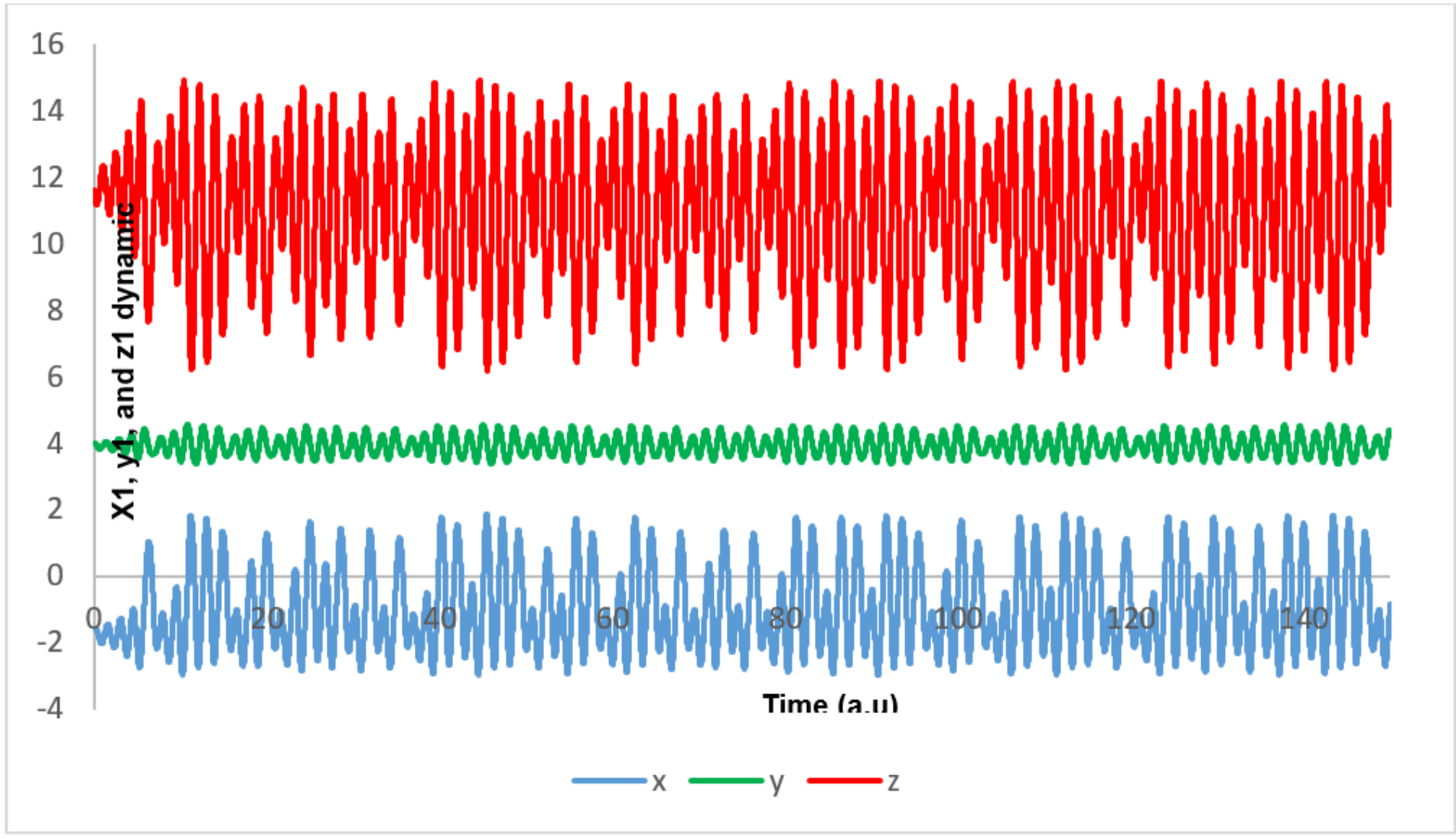

Figure 8

Time series in $x 1, y 1$ and $z 1$-dynamics of Chua system with new scheme. 


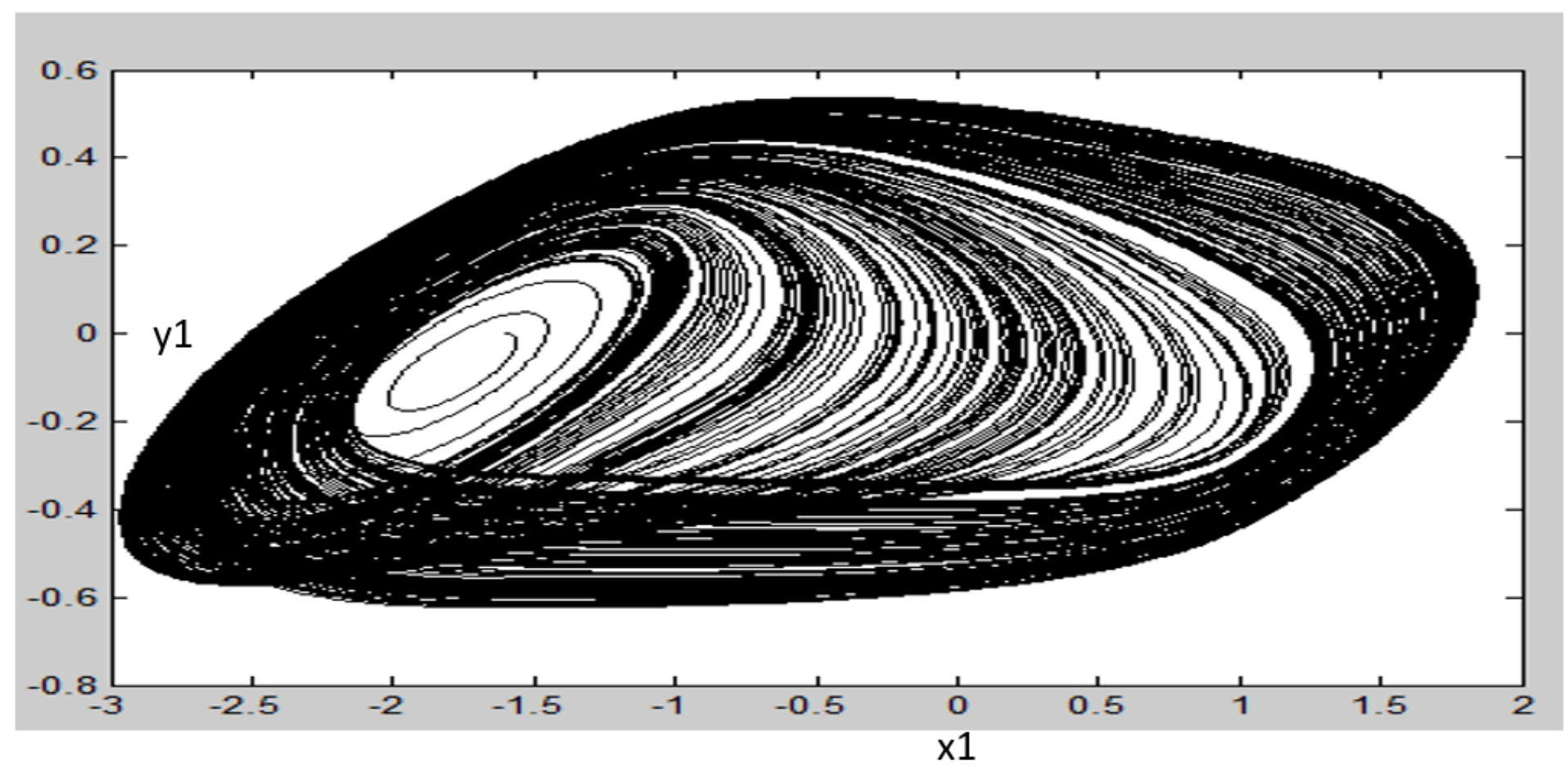

Figure 9

Strange attractor in (y1-x1) dynamics of Chua system with new scheme.

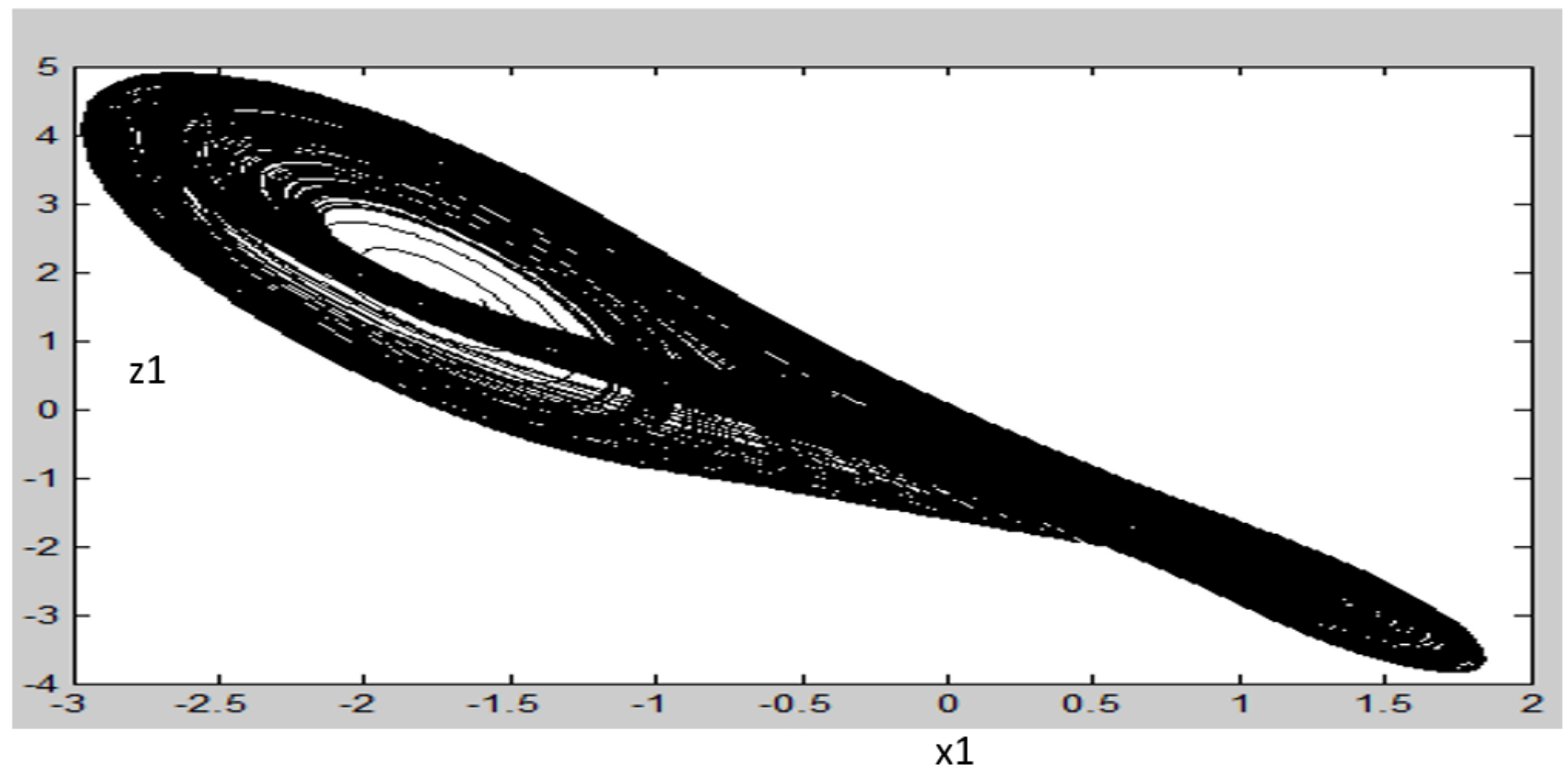

Figure 10

Strange attractor in (z1-x1) dynamics of Chua system with new scheme. 


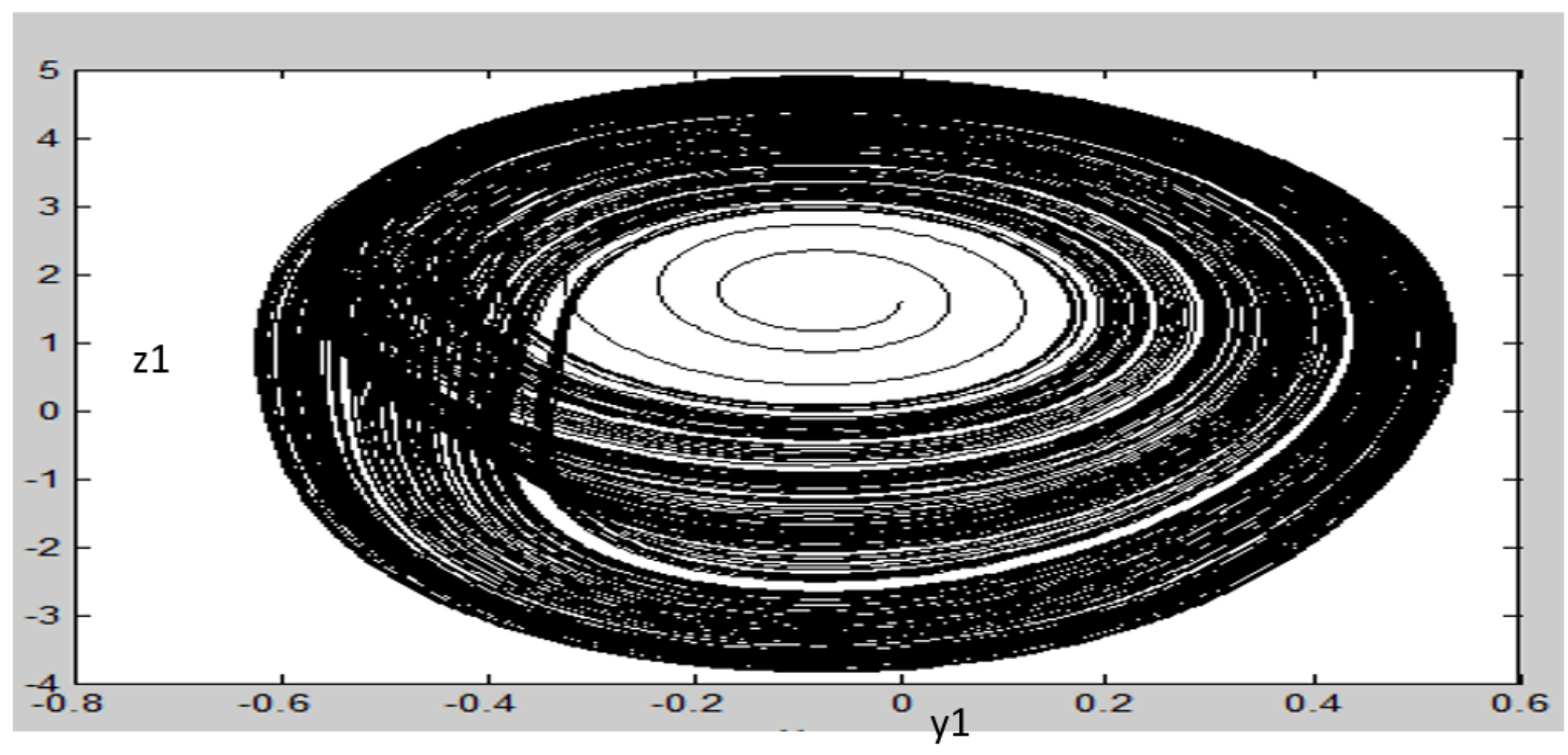

Figure 11

Strange attractor in (z1-y1) dynamics of Chua system with new scheme.

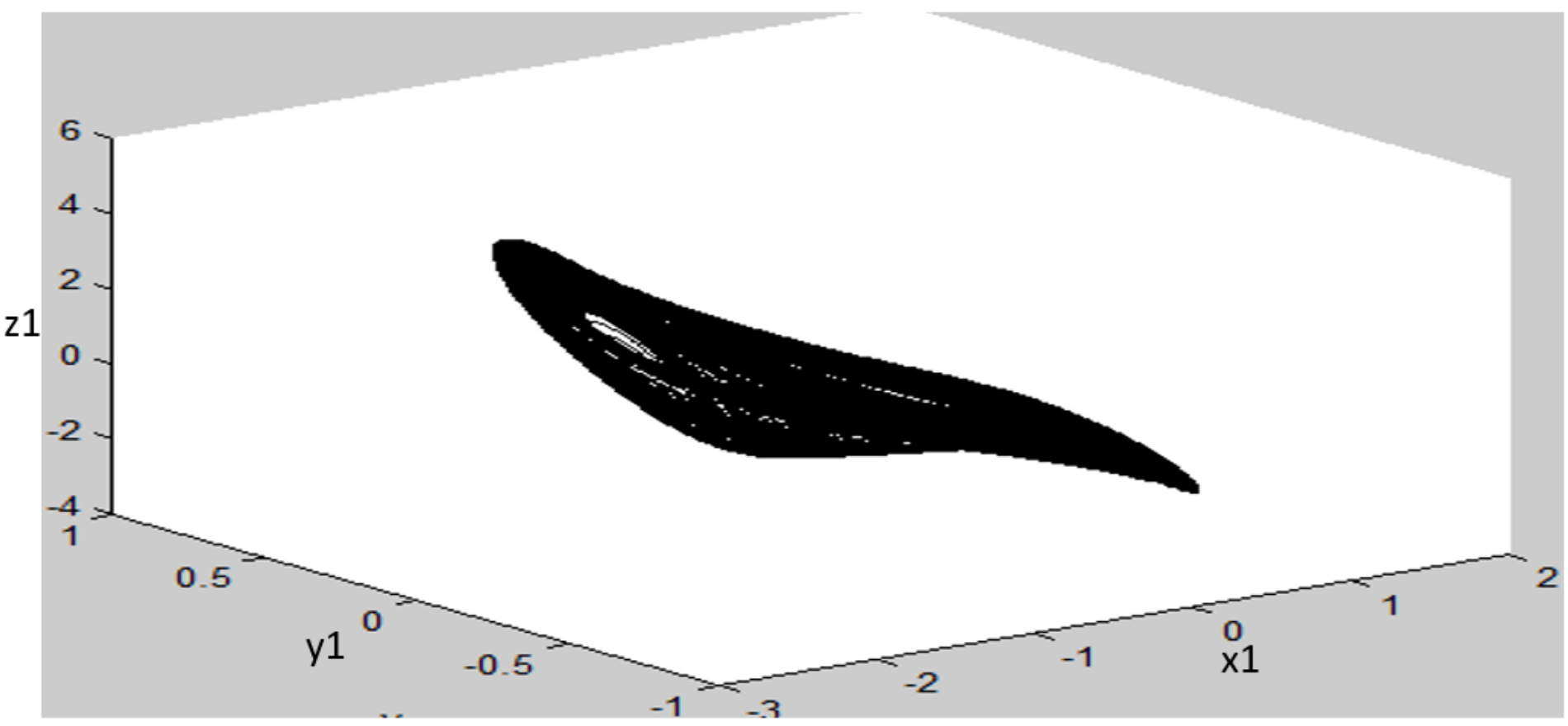

Figure 12

Strange attractor in (z1-y1-x1) dynamics of Chua system with new scheme. 


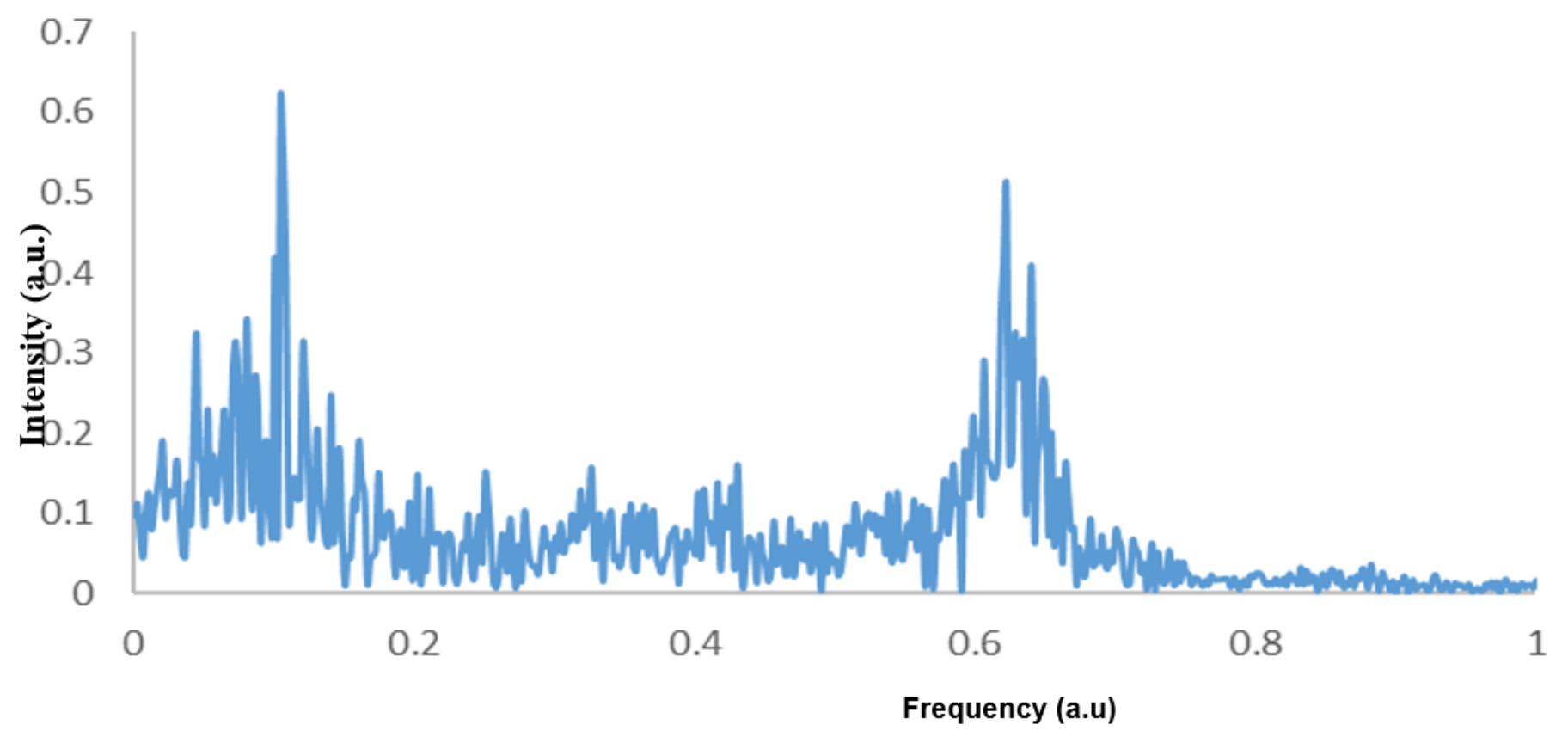

Figure 13

FFT spectrum of z-dynamics of Chua system.

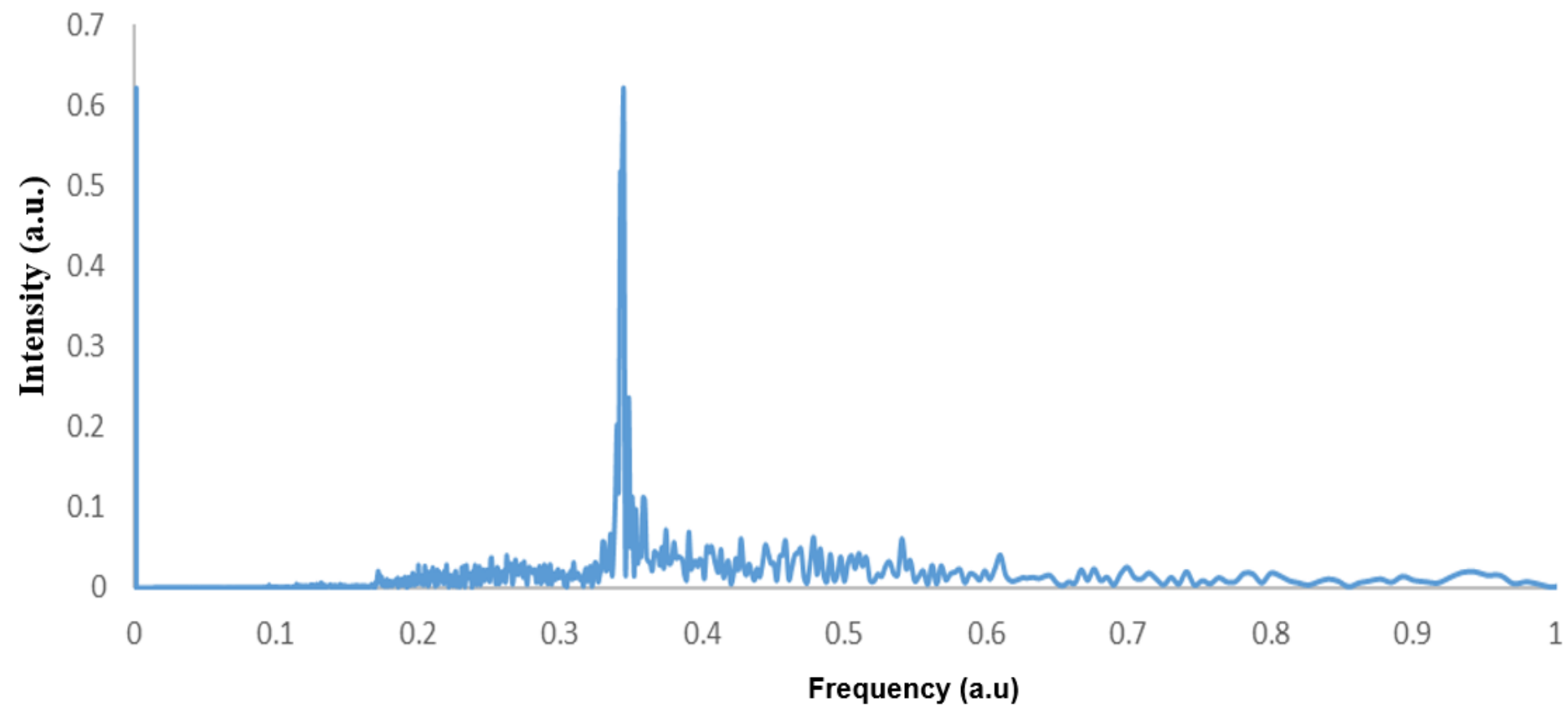

Figure 14

FFT specrum of z-dynamics of Chua system with new scheme . 